\section{TEXTURE ANALYSIS OF IRIS BIOMETRICS BASED ON ADAPTIVE SIZE NEIGHBORHOOD ENTROPY AND LINEAR DISCRIMINANT ANALYSIS}

\author{
Saša Adamović ${ }^{1}$, Aleksandar Savić2 ${ }^{2}$ Milan Milosavljević ${ }^{1}$, Slađana Spasić \\ ${ }^{1}$ Singidunum University, Serbia \\ ${ }^{2}$ University of Belgrade
}

\begin{abstract}
:
Novel method for personal identification, based on adaptive size neighborhood entropy of iris images, was proposed. Through the process of segmentation, iris was extracted from other regions of the human eye, geometrically transformed and normalized. Entropy calculations performed for different neighborhood sizes allows simultaneous distinguishing of fine and global iris texture. Described method also allows recognition of images which contain artifacts and their removal from further analysis after application of principal component analysis (PCA). In the last analytical step, linear discriminant analysis (LDA) with training vector set was applied, allowing rigorous classification. Described procedure is suitable for the application in security systems with small number of authorized persons and a high degree of safety.
\end{abstract}

\section{INTRODUCTION}

Biometric systems are used for identifying based on physical or behavioral characteristics of human beings, such as face, voice, fingerprint and iris. Biometric data has the potential advantage of becoming a unique identifier of a person. Depending on the type of biometric samples may contain a sufficient amount of information difficult to uncover. Iris biometrics is imposed as a possible solution because of its uncertainty and uniqueness properties. To obtain the maximum of information from the iris, it is necessary to know the details of the extraction process and the different types of noise that affect the process of generating a biometric template.

Local entropy was determined by the region of iris image [1]. The resolution of the segmented iris was $240 \times 20$ pixels. Iris was divided into two regions, inner and outer. Inner region is positioned between the pupil and the outer region of iris which is located between the corneal and internal region (Figure 1). According to literature [3], inner region has significantly higher local entropy. Maximum entropy values for the neighborhood size $9 \times 9$ pixels were: in the inner region 4.4412 bits/pixel, and 3.6020 bits/pixel in the outer region.

\section{Key words:}

Biometrics, entropy,

iris,

linear discriminant analysis (LDA), texture analysis.

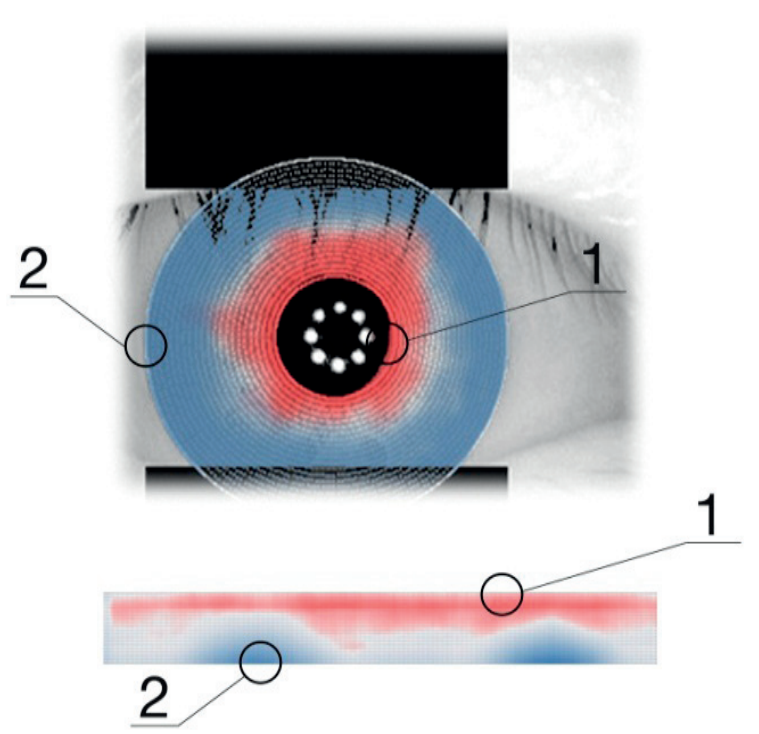

Fig. 1. Entropy of the texture of Iris by region. Upper panel: 1 - Inner region positioned close to pupil. 2 - Outer region positioned close to cornea. Lower panel: The projection of the iris onto a rectangle. 
Figure 1 clearly illustrates the extent of the value of the entropy. Iris texture colored in red indicate increased entropy, and part painted blue indicates a significant decrease in the entropy of iris texture.

Texture analysis based on entropy has found application in medicine for magnetic resonance images, in material science and pattern recognition $[2,3]$.

Aim of the analysis was to create analytical procedure that could be applied for recognition of small number of persons (up to 10), such as banks.

Images of iris could be affected with artifacts, thus multiple images should be captured for each person. This step is not expensive or time consuming, therefore 10 or more images could be captured, and those which contain artifacts excluded from further analysis.

Graphical representation of analytical procedure is presented in Figure 2.

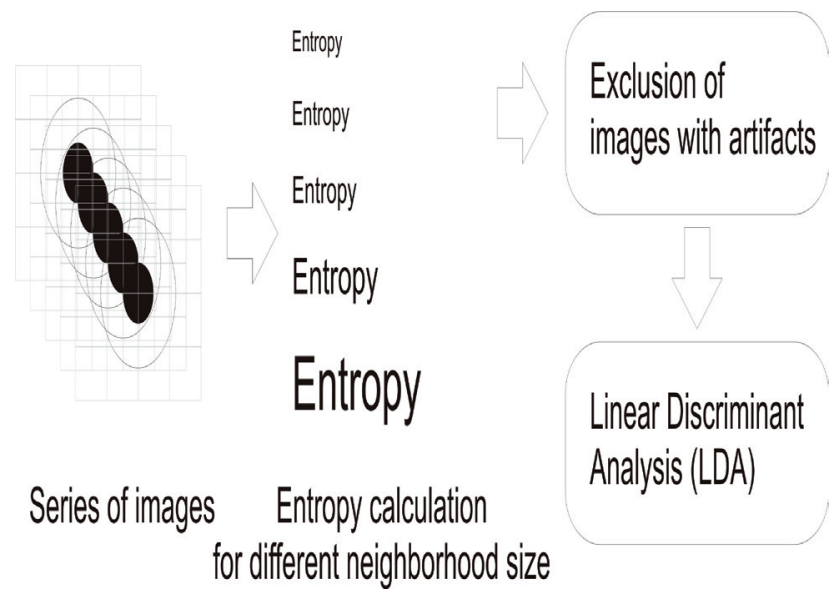

Fig. 2. Scheme of analytical procedure.

\section{MATERIALS AND METHODS}

\section{Obtaining iris images from database}

To test the system, data sets of eye images were used as inputs. For the analysis 10 persons, each represented with 10 images, were randomly chosen from CASIA-IrisV4 database of 54,607 grayscale eye images courtesy of The Chinese Academy of Sciences - Institute of Automation (CASIA).

The first task was to determine localization of iris, which provides its insulation from other regions of the human eye from raw digital photos. The process is very complex, but crucial for further success of the analysis. If poorly positioned in a circle around the iris, segmentation process will be unsuccessful which is further reflected in poor authentication or increased false reject rate (FRR) parameter.

The algorithm uses Hough transformation [4] in order to determine the parameters of a geometric object, in this case a circle that describes the location of the iris. In addition to the localization algorithm, the performance of the segmentation process depends on the quality of iris image. Normalized iris localization process (Figure 3) provides the output image which will be used as template for calculating the local entropy. Radial parameter for normalization is 20 pixels and the angle parameter for normalization is 240 pixels. Total normalized iris resolution is $240 \times 20$ pixels.

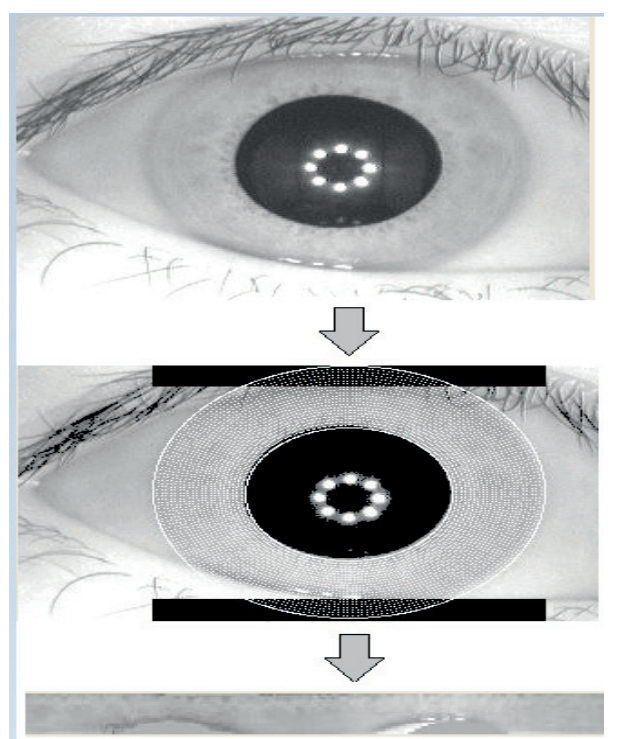

Fig. 3. Process of segmentation. Radial parameter for normalization was 20 pixels, and the angle parameter for normalization was 240 pixels.

\section{Entropy calculations}

Entropy represents measure of randomnes, thus any imperfections in image will be recognised. Entropy was calculated according to equation 1 :

$$
\text { Entropy }=-\sum_{i=1}^{N g} \sum_{j=1}^{N g} P(i, j) \log P(i, j)
$$

where $P$ represents the histogram counts from the defined neighbourhood for the given pixel on coordinate $(i, j)$.

Entropy has been calculated for the different circular neighbourhood sizes (from 2 to 5 pixels), therefore fine and global texture of iris were examined simultaneously. Histograms were constructed for each entropy image, and merged to build larger vector

$$
v_{1 \times 20}^{1} \ldots v_{1 \times 20}^{5} \rightarrow v_{1 \times 100}^{t}
$$

Optimal number of beans is between 15 and 25, in our case was 20. Entropy calculations were performed in self developed MatWorks, MatLab 2010a based code.

\section{Linear discriminant analysis (LDA)}

The linear discriminant analysis (LDA) is a representative of the supervised learning method. LDA has achieved superior classification results in comparison with principal component analysis (PCA) for the observed data sets. However, PCA can be successfully implemented for exclusion of artefact images.

Let the $\mathbf{T}$ be the matrix of all the training vectors (textural results obtained from irises). Scatter matrices 
within-class $\mathbf{S}_{W}$ and between-class $\mathbf{S}_{\mathbf{B}}$ can be described as (equation 3 and 4 ):

$$
\begin{aligned}
& \mathrm{S}_{\mathrm{w}}=\sum \mathrm{S}_{\mathrm{y}} \\
& \mathrm{S}_{\mathrm{y}}=\sum\left(\mathrm{x}_{\mathrm{i}}-\mu_{\mathrm{i}}\right)\left(\mathrm{x}_{\mathrm{i}}-\mu_{\mathrm{i}}\right)^{\top} \\
& \mathrm{S}_{\mathrm{B}}=\sum\left|I_{y}\right|\left(\mu_{\mathrm{y}}-\mu\right)\left(\mu_{\mathrm{y}}-\mu\right)^{\top}
\end{aligned}
$$

$\boldsymbol{\mu}$ - denotes the total mean vector, and the $\boldsymbol{\mu}_{\mathrm{y}}$ stands for the class mean vector. Vectors $\boldsymbol{\mu}$ and $\boldsymbol{\mu}_{\mathrm{y}}$ were defined as (equation 5):

$$
\mathbf{i}=\frac{1}{l} \sum \mathbf{x}_{\mathbf{i}} \quad \mathbf{i}_{y}=\frac{1}{\left|I_{y}\right|} \sum \mathbf{x}_{\mathbf{i}}
$$

Objective of LDA is to train the linear data projection

$$
z=\mathbf{W}^{T} x
$$

Such the class separability is maximized. Criterion for the separability is defined as (equation 6)

$$
F(\mathbf{W})=\frac{\operatorname{det}\left(\mathbf{S}_{\mathbf{B}}\right)}{\operatorname{det}\left(\mathbf{S}_{\mathbf{W}}\right)}
$$

LDA analyses was performed in STPRtool for MatLab 2010a.

\section{RESULTS AND DISCUSSION}

First analytical step requires entropy histogram calculations (Figure 4).

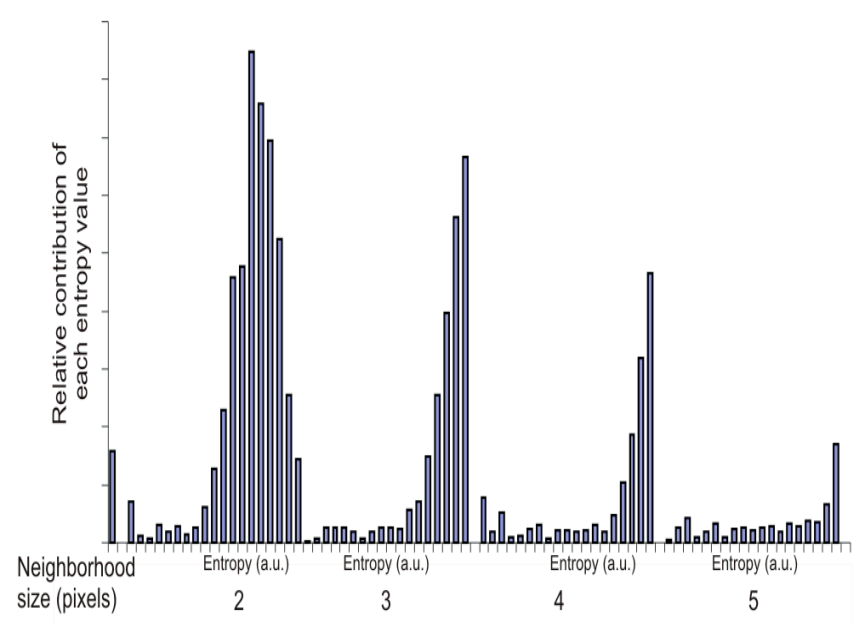

Fig. 4. Typical entropy histogram. Number of beans for each neighborhood was 20 .

Next step involves exclusion of images containing artifacts through application of principal component analysis (PCA). Depending on person, 1 to 4 images from 10 were rejected from the further analysis.

LDA has prove the efficiency leading to very strict classification, thus distance between members of single class were several orders of magnitude smaller than distances between different classes (Figure 5).

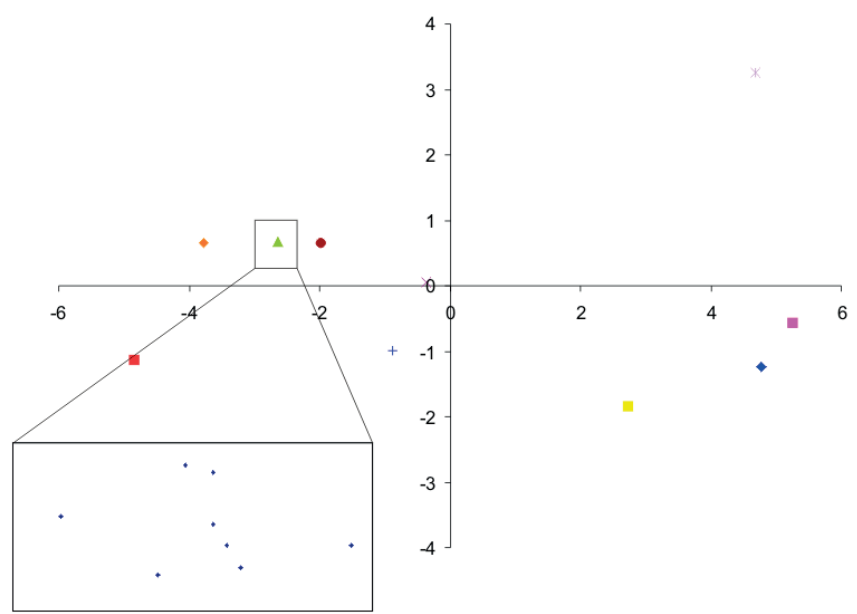

Fig. 5. LDA scores for personal identification by using adaptive size neighborhood entropy. Each dot represents single person. Different images of the same person are represented with the small dots in the rectangle. Distances between the classes were several orders of magnitude larger than between the members of individual classes.

\section{CONCLUSION}

Proposed novel method has provided excellent possibilities for personal identification if applied for small groups. Distances between different classes (different persons) were so much greater comparing to distances between members of individual classes (different images of the same person) that possibility of false recognition was very low.

Calculations of statistical parameters for different neighborhood sizes are promising and easy to implement in the existing analyses.

Described analytical procedure could be improved if the images of better resolution were used, or if additional classification methods such as support vector machine (SVM) were used.

\section{Acknowledgment}

This work was supported by projects OI 173017, 173045, III41026, TR32054 financed by The Serbian Ministry of Education and Science.

\section{REFERENCES}

[1] S. Adamović, M. Milosavljević, "Theoretical analysis of iris biometrics information for extraction cryptology keys", Society for Electronics, Telecommunications, Computers, Automatic Control and Nuclear Engineering, Etran 2012.

[2] S. Watanabe, Pattern recognition as a quest for minimum entropy, Pattern Recogn., vol. 13, 1981, p.p. 381-387.

[3] V. A. Kovalev, F. Kruggel, H.-J. Grtz, D. Y. von Cramon, Three-dimensional texture analysis of MRI brain datasets, Med. imag., IEEE, 2001, vol. 20, 2001, p.p. 424-433.

[4] D. J. Kerbyson, and T. J. Atherton, "Circle detection using Hough transform filters", Fifth International Conference on Image Processing and its Applications, 1995, p.p. 370-374. 